\title{
Astaxanthin reduces isoflurane-induced neuroapoptosis via the PI3K/Akt pathway
}

\author{
CHUN-MEI WANG, XIAO-LAN CAI and QING-PING WEN
}

Department of Anesthesiology, The First Affiliated Hospital of Dalian Medical University, Dalian, Liaoning 116011, P.R. China

Received April 20, 2015; Accepted January 25, 2016

DOI: $10.3892 / \mathrm{mmr} .2016 .5035$

\begin{abstract}
Astaxanthin is an oxygen-containing derivative of carotenoids that effectively suppresses reactive oxygen and has nutritional and medicinal value. The mechanisms underlying the effects of astaxanthin on isoflurane-induced neuroapoptosis remain to be fully understood. The present study was conducted to evaluate the protective effect of astaxanthin to reduce isoflurane-induced neuroapoptosis and to investigate the underlying mechanisms. The results demonstrated that isoflurane induced brain damage, increased caspase-3 activity and suppressed the phosphatidylinositol 3-kinase $(\mathrm{PI} 3 \mathrm{~K}) /$ protein kinase B (Akt) signaling pathway in an in vivo model. However, treatment with astaxanthin significantly inhibited brain damage, suppressed caspase-3 activity and upregulated the PI3K/Akt pathway in the isoflurane-induced rats. Furthermore, isoflurane suppressed cell growth, induced cell apoptosis, enhanced caspase-3 activity and downregulated the PI3K/Akt pathway in organotypic hippocampal slice culture. Administration of astaxanthin significantly promoted cell growth, reduced cell apoptosis and caspase-3 activity, and upregulated the PI3K/Akt pathway and isoflurane-induced neuroapoptosis. The present study demonstrated that downregulation of the PI3K/Akt pathway reduced the effect of astaxanthin to protect against isoflurane-induced neuroapoptosis in the in vitro model. The results of the current study suggested that the protective effect of astaxanthin reduces the isoflurane-induced neuroapoptosis via activation of the PI3K/Akt signaling pathway.
\end{abstract}

\section{Introduction}

Researchers in the field of anesthesia are actively investigating inhalation anesthetics due to their rapid effects, protective effect against ischemic injury of vital organs, including the

Correspondence to: Mr. Chun-Mei Wang, Department of Anesthesiology, The First Affiliated Hospital of Dalian Medical University, 222 Zhongshan Road, Dalian, Liaoning 116011, P.R. China

E-mail: chunmeiwangcmx@163.com

Key words: astaxanthin, isoflurane, neuroapoptosis, PI3K/Akt heart, brain and kidneys, and possible neurotoxicity (1). Previous in vitro and in vivo studies at a molecular and cellular level suggested that numerous ion channel receptors and classical neurotransmitters may be potential molecular targets of general anesthetics (2-4). Evidence regarding the neurotoxicity of inhalation anesthetics are primarily from animal studies, and the mechanism of activation has been investigated in certain experimental studies focussing upon apoptosis (4-6).

Currently used inhalation anesthetics include enflurane, halothane, isoflurane, sevoflurane, desflurane and nitrous oxide $(2,7)$. Isoflurane is a structural isomer of enflurane, and a colorless transparent liquid with a pungent aroma of ether (1). Isoflurane is widely used as an inhalation anesthetic in the clinic due to chemical stability, high efficiency, ease of regulating the depth of anesthesia, low metabolic rate and excretion of the liver, low toxicity to the kidneys and rapid, significant clinical effects (7). Numerous anesthesiologists consider it as the first choice of inhalational anesthetics (2). Previous clinical applications suggested that the effect of isoflurane on the nervous system was beneficial (1). Compared with other anesthetics, isoflurane suppresses the increase of blood flow to the brain $(7,8)$, thus it may be used for brain surgery. Furthermore, the superficial anesthesia of isoflurane does not affect the brain waves of patients. Whether the anesthesia is repeated or extended, it has been reported that it will not lead to lasting dysfunction of the central nervous system (5).

Natural astaxanthin is a type of lutein, obtained by oxidation of carotenoids, and it is abundant in certain species, such as salmon and shellfish (9). Previous studies investigated the chemical structure and excellent performance of astaxanthin in anti-oxidation, anti-inflammatory, antitumor, anti-Helicobacter pylori infection and anti-UV processes $(10,11)$. Whether astaxanthin reduces the isoflurane-induced neuroapoptosis through the phosphatidylinositol 3-kinase (PI3K)/protein kinase B (Akt) pathway is undetermined. The aim of the present study was to elucidate whether astaxanthin reduces isoflurane-induced neuroapoptosis, utilizing validated in vivo and in vitro models, and to explore its possible mechanisms.

\section{Materials and methods}

In vivo model experiments and grouping. A total of 30 male Sprague-Dawley rats (weight, 250-300 g; age, 7 days) were 
purchased from the Experimental Center of Dalian Medical University (Dalian, China), and were maintained at $22-25^{\circ} \mathrm{C}$ and 40-50\% humidity, under a 12-h light/dark cycle with ad libitum access to food and water. The rats were randomly assigned into three groups as follows: i) Control group, rats were administered saline by intraperitoneal injection (i.p.); ii) model group, rats were exposed to $0.75 \%$ isoflurane (Sigma-Aldrich, St. Louis, MO, USA) for $6 \mathrm{~h}$ in $25 \%$ oxygen or air in a temperature-controlled chamber, and administered saline (i.p.); and iii) treated group, randomly assigned rats from the model group were further administered astaxanthin (100 mg/kg/day, i.p.; 97\% purity; Sigma-Aldrich). All groups were treated for 7 days. Arterial blood gases and glucose levels in each group were detected using an automated biochemistry analyzer (SMT100V; Robonik India Pvt. Ltd., Maharashtra, India) at the Clinical Laboratory of The First Affiliated Hospital of Dalian Medical University. The present study was approved by the ethics committee of The First Affiliated Hospital of Dalian Medical University.

Quantitative histology of in vivo neurodegeneration in isofluorane-induced rats. An optical dissector and fractionator method (Stereo Investigator System; MBFBioscience, Williston, VT, USA) was utilized to measure neurodegeneration in the rat hippocampi. Briefly, following anesthetization with $1.5 \%$ sodium pentobarbital (Sigma-Aldrich), the rats were sacrificed by decollation and the rat hippocampi were harvested. A counting frame $(0.05 \times 0.05 \times 0.05 \mathrm{~mm})$ and a high numerical aperture objective lens were used to visualize the hippocampi neurons. Sampling of the hippocampus was performed by randomly selecting 10-15 viewing fields for which the counting frame was positioned to count at different focal levels (Stereo Investigator System; Microbright Field, Williston, VT, USA).

Measurement of caspase-3 activity. Tissue and cell samples were dissociated with 10 volumes of tissue lysis buffer (Beyotime Institute of Biotechnology, Haimen, China) and centrifuged at $12,300 \mathrm{x}$ g for $10 \mathrm{~min}$ at $4^{\circ} \mathrm{C}$. Protein concentrations were measured using the bicinchoninic acid (BCA) protein assay kit (Bio-Rad Laboratories, Inc., Hercules, CA, USA) in accordance with the manufacturer's instructions. Cleavage of chromogenic caspase substrate Ac-DEVD-pNA and equal protein were mixed in accordance to manufacturer's instructions (Promega Corporation, Madison, WI, USA), and used to measure caspase-3 activity at $405 \mathrm{~nm}$ optical density with a spectrophotometer (BioTek Synergy ${ }^{\mathrm{TM}}$ Microplate Reader; BioTek Instruments, Inc., Winooski, VT, USA).

Western blot analysis of phosphorylated ( $p)$-Akt and Akt protein expression. Proteins were extracted and quantified using the BCA protein assay kit as described above. Equal amounts of protein $(50 \mu \mathrm{g})$ were separated by $10 \%$ sodium dodecyl sulfate-polyacrylamide gel electrophoresis and transferred to polyvinyl difluoride membranes (Amersham; GE Healthcare Life Sciences, Chalfont, UK). Membranes were blocked with $5 \%$ non-fat milk diluted in phosphate-buffered saline (PBS), and then were incubated with rabbit anti-p-Akt (sc-33437; 1:2,000), anti-Akt (sc-8312; 1:1,000; both Santa Cruz Biotechnology, Inc., Dallas, TX, USA) and anti- $\beta$-actin (D110007; 1:500; Sangon Biotech Co., Ltd., Shanghai, China) polyclonal antibodies overnight at $4^{\circ} \mathrm{C}$. Membranes

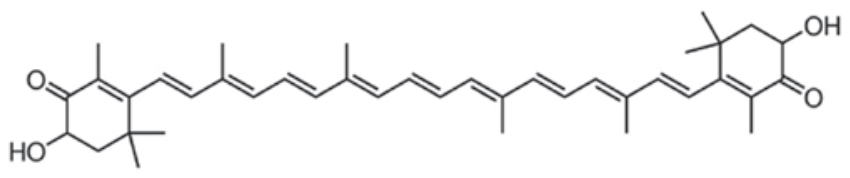

Figure 1. The chemical structure of astaxanthin.

were then washed with Tris-buffered saline and Tween-20 (Sangon Biotech Co., Ltd.) for $2 \mathrm{~h}$ at room temperature, and incubated with horseradish peroxidase-conjugated secondary antibody (SN134; 1:5,000; Sunshine Biotechnology Co., Ltd., Nanjing, China) for $2 \mathrm{~h}$ at room temperature. The membranes were incubated with enhanced chemiluminescence reagent (Amersham; GE Healthcare Life Sciences), and protein expression was analyzed using ImageQuant TL software, version 2003.03 (GE Healthcare Life Sciences).

In vitro model experiments and grouping. A total of 24 male C57Bl/6 mice (age, 7-8 weeks; weight, 250-300 g) were obtained from the SPF Animal Experiment Center of Dalian Medical University. The mice were maintained at $22 \pm 1^{\circ} \mathrm{C}$ and $50-60 \%$ humidity under a $12-\mathrm{h}$ light/dark cycle. The mice were sacrificed by cervical dislocation following anesthetization with $40 \mathrm{mg} / \mathrm{kg}$ sodium pentobarbital, after which organotypic hippocampal tissue was immediately harvested and seeded into $25 \mathrm{~cm}^{2}$ plastic bottles to separate the cells. Cells were cultivated in Dulbecco's modified Eagle's medium (DMEM; Thermo Fisher Scientific, Inc., Waltham, MA, USA) containing $10 \%$ fetal bovine serum (FBS; Gibco; Thermo Fisher Scientific, Inc.), for $24 \mathrm{~h}$ in a humidified atmosphere enriched with $5 \% \mathrm{CO}_{2}$, at $37^{\circ} \mathrm{C}$. Following a 24-h cultivation, non-adherent cells were discarded and $0.25 \%$ trypsin (Sunshine Biotechnology Co., Ltd.) was utilized to transfer adherent cells into $25 \mathrm{~cm}^{2}$ plastic bottles. Cells were cultivated with DMEM/F-12 (Thermo Fisher Scientific, Inc.) supplemented with $10 \%$ FBS and $1 \%$ penicillin/streptomycin (Sunshine Biotechnology Co., Ltd.) at $37^{\circ} \mathrm{C}$ in a humidified atmosphere. Organotypic hippocampal cells were separated into four groups ( $n=6)$ as follows: i) Control, untreated; ii) model group, the cells were treated with $0.75 \%$ isoflurane $+50 \mu \mathrm{M}$ gabazine (Sigma-Aldrich) for $24 \mathrm{~h}$; iii) treated, the cells were treated with $0.75 \%$ isoflurane $+50 \mu \mathrm{M}$ gabazine $+8 \mu \mathrm{M}$ astaxanthin for $24 \mathrm{~h}$; iv) treated + LY294002 (LP), the cells were treated with $20 \mu \mathrm{M}$ LP (Sigma-Aldrich) $+0.75 \%$ isoflurane $+50 \mu \mathrm{M}$ gabazine $+8 \mu \mathrm{M}$ astaxanthin for $24 \mathrm{~h}$.

Measurement of cell growth using MTT. Organotypic hippocampal cells were seeded into 96-well plates at a density of $1.5 \times 10^{3}$ cells/well. MTT (10 $\mu \mathrm{l}$; Sangon Biotech Co., Ltd.) was added to the cells and incubated for $4 \mathrm{~h}$ in a humidified atmosphere enriched with $5 \% \mathrm{CO}_{2}$ at $37^{\circ} \mathrm{C}$. Dimethyl sulfoxide (150 $\mu \mathrm{l}$; Invitrogen; Thermo Fisher Scientific, Inc.) was added to each well and plates were shaken for $20 \mathrm{~min}$ at room temperature. Absorbance was measured at $450 \mathrm{~nm}$ with a microplate reader (R\&D Systems, Inc., Minneapolis, MN, USA).

Measurement of cell apoptosis using flow cytometry. Organotypic hippocampal cells were seeded into 6-well plates at a density of $1-2 \times 10^{6}$ cells/well. Cells were then washed twice 
A

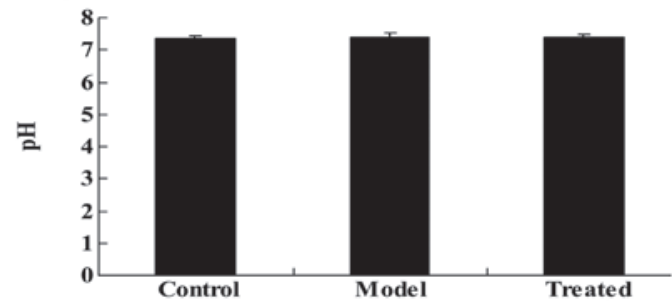

B

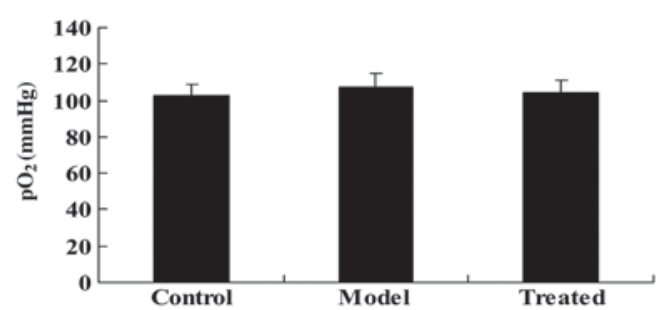

C

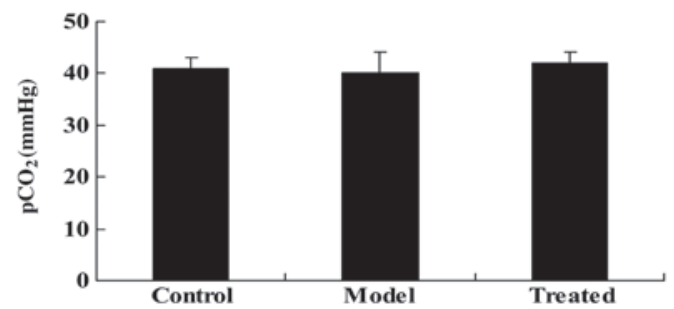

D

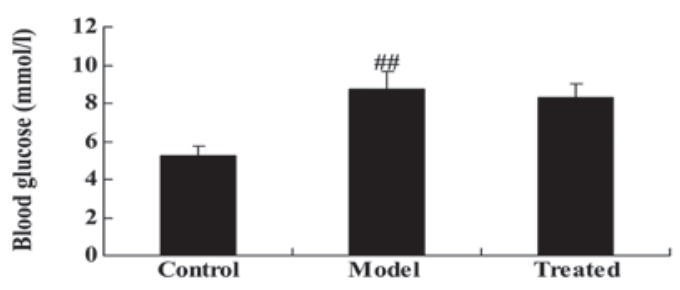

Figure 2. The effect of astaxanthin on arterial blood gases (A) $\mathrm{pH},(\mathrm{B}) \mathrm{pO}_{2}$ and (C) $\mathrm{pCO}_{2}$, and (D) on blood glucose levels in untreated (control), isoflurane-treated (model) and astaxanthin + isoflurane-treated (treated) rats. ${ }^{\# \#} \mathrm{P}<0.01$ vs. the control group. $\mathrm{pO}_{2}$, partial pressure of oxygen; $\mathrm{pCO}_{2}$, partial pressure of carbon dioxide; model, isoflurane-induced; treated, astaxanthin-treated.

with ice-cold PBS, and $50 \mu 1$ lysis buffer was added to each well. Annexin V-fluorescein isothiocyanate $(5 \mu \mathrm{l})$ and propidium iodide ( $5 \mu \mathrm{l}$; BD Biosciences, San Jose, CA, USA) staining was performed, according to the manufacturer's instructions. Flow cytometry (FACScan; BD Biosciences) and CellQuest Pro software, version 5.1 (BD Biosciences) were used to analyze cell apoptosis.

Statistical analysis. Results were analyzed with SPSS software, version 17 (SPSS, Inc., Chicago, IL, USA) using one-way analysis of variance, followed by Dunnett's post hoc test. Data are expressed as the mean \pm standard deviation. $\mathrm{P}<0.05$ was considered to indicate a statistically significant difference.

\section{Results}

Effect of astaxanthin on arterial blood gases and glucose levels. The chemical structure of astaxanthin is presented in Fig. 1. As demonstrated in Fig. 2A-C, in vivo experiments

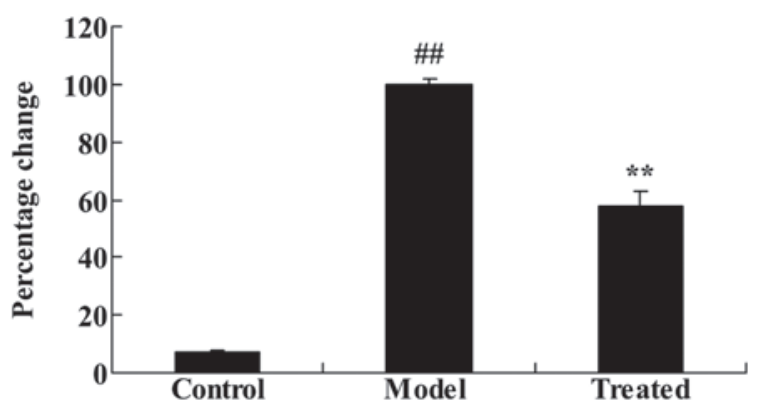

Figure 3. The effect of astaxanthin on brain damage severity in untreated (control), isoflurane-treated (model) and astaxanthin + isoflurane-treated (treated) rats. ${ }^{\# \#} \mathrm{P}<0.01$ vs. the control group; ${ }^{* *} \mathrm{P}<0.01$ vs. the model group. Model, isoflurane-induced; treated, astaxanthin-treated.

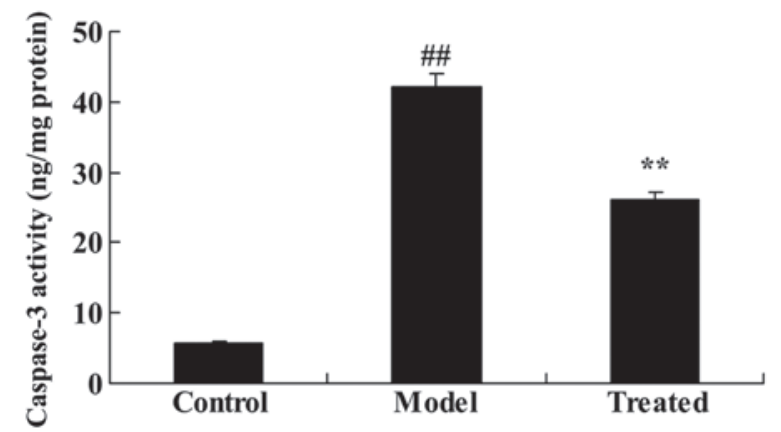

Figure 4. The effect of astaxanthin on caspase-3 activity in untreated (control), isoflurane-treated (model) and astaxanthin + isoflurane-treated (treated) rats. ${ }^{\#} \mathrm{P}<0.01$ vs. the control group and ${ }^{* * *} \mathrm{P}<0.01$ vs. the model group. Model, isoflurane-induced; treated, astaxanthin-treated.

detected no significant differences among groups for arterial blood gases ( $\mathrm{pH}, \mathrm{pO}_{2}$ and $\mathrm{pCO}_{2}$ ). Blood glucose levels were significantly increased in the model group compared with the control group $(\mathrm{P}<0.01)$, and markedly increased in the astaxanthin-treated group (Fig. 2D).

Astaxanthin protects against isoflurane-induced brain damage in Sprague-Dawley rats. As demonstrated in Fig. 3, administration of isoflurane significantly increased the rate of neuronal cell apoptosis compared with the control group $(\mathrm{P}<0.01)$. Additional treatment with astaxanthin significantly reduced the isoflurane-induced brain damage, compared with the model group $(\mathrm{P}<0.01$; Fig. 3).

The effect of astaxanthin on caspase-3 activity in isoflurane-treated rats. As demonstrated in Fig. 4, caspase-3 activity was significantly increased following isoflurane treatment, compared with the control group $(\mathrm{P}<0.01)$. Further treatment with astaxanthin significantly suppressed the isoflurane-induced caspase-3 activity, compared with the model group ( $\mathrm{P}<0.01$; Fig. 4).

The effect of astaxanthin on the PI3K/Akt signaling pathway in isoflurane-treated rats. To determine whether astaxanthin protects against isoflurane-induced brain damage in rats, p-Akt and Akt protein expression levels were determined using western blotting. The results demonstrated that the 
A

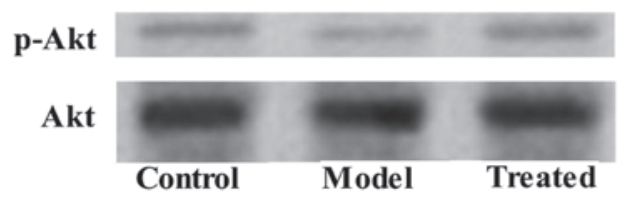

B

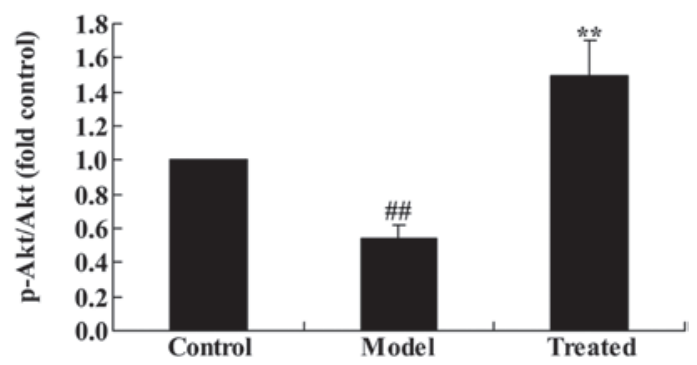

Figure 5. The effect of astaxanthin on the p-Akt protein expression levels in untreated (control), isoflurane-treated (model) and astaxanthin + isoflurane-treated (treated) rats. (A) Western blotting assay and (B) statistical analysis of the $\mathrm{p}$-Akt/Akt ratio. ${ }^{\# \#} \mathrm{P}<0.01 \mathrm{vs}$. the control group and ${ }^{* *} \mathrm{P}<0.01$ vs. the model group. $\mathrm{p}$-Akt, phosphorylated protein kinase $\mathrm{B}$; model, isoflurane-induced; treated, astaxanthin-treated.

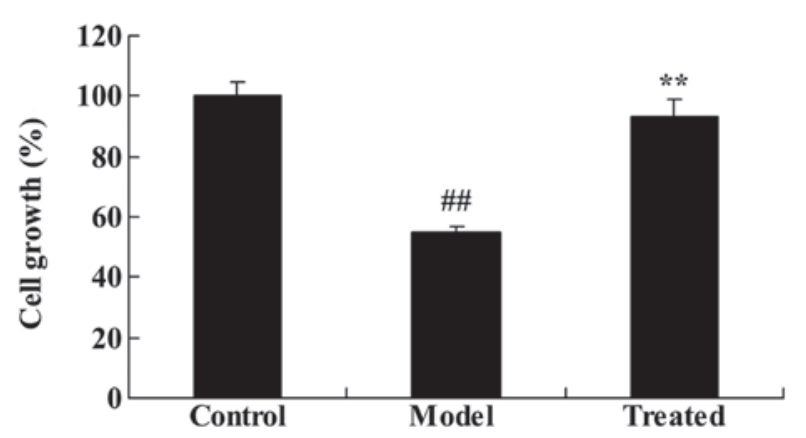

Figure 6. The effect of astaxanthin on the cell growth in untreated (control), isoflurane-treated (model) and astaxanthin + isoflurane-treated (treated) rats. ${ }^{\# /} \mathrm{P}<0.01$ vs. the control group and ${ }^{* *} \mathrm{P}<0.01$ vs. the model group. Model, isoflurane-induced; treated, astaxanthin-treated.

p-Akt/Akt ratio was significantly downregulated in the isoflurane-treated group compared with the control group $(\mathrm{P}<0.01$; Fig. 5). Additional treatment with astaxanthin significantly upregulated the effect of isoflurane treatment compared with the model group $(\mathrm{P}<0.01$; Fig. 5$)$.

The effect of astaxanthin on the cell growth of isoflurane-treated rats. As demonstrated in Fig. 6, the in vitro experiments indicated that isoflurane treatment significantly reduced organotypic hippocampal cell growth, compared with the control group $(\mathrm{P}<0.01)$. Further treatment with astaxanthin significantly reversed the isoflurane-suppressed cell growth, compared with the model group.

The effect of astaxanthin on the cell apoptosis of isoflurane-treated rats. As demonstrated in Fig. 7 the in vitro experiments indicated that treatment with isoflurane significantly increased cell apoptosis, compared with the control group $(\mathrm{P}<0.01)$. Administration of astaxanthin significantly reduced the effect of isoflurane-treatment, compared with the model group ( $\mathrm{P}<0.01 ;$ Fig. 7$)$.

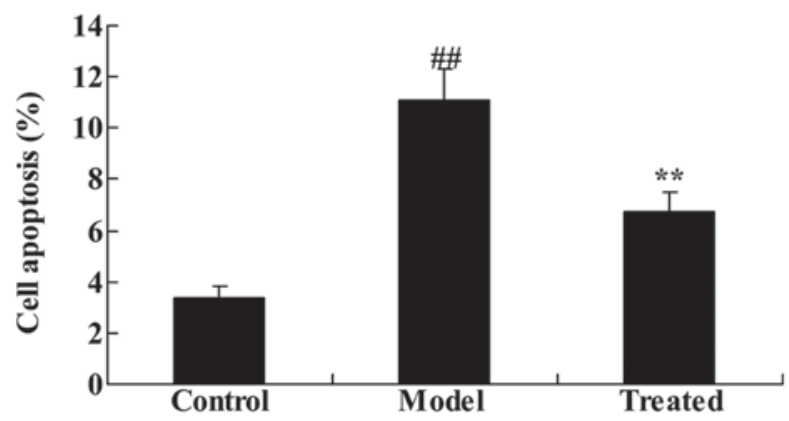

Figure 7. The effect of astaxanthin on cell apoptosis in untreated (control), isoflurane-treated (model) and astaxanthin + isoflurane-treated (treated) rats. ${ }^{\# \#} \mathrm{P}<0.01$ vs. the control group and ${ }^{* *} \mathrm{P}<0.01$ vs. the model group. Model, isoflurane-induced; treated, astaxanthin-treated.

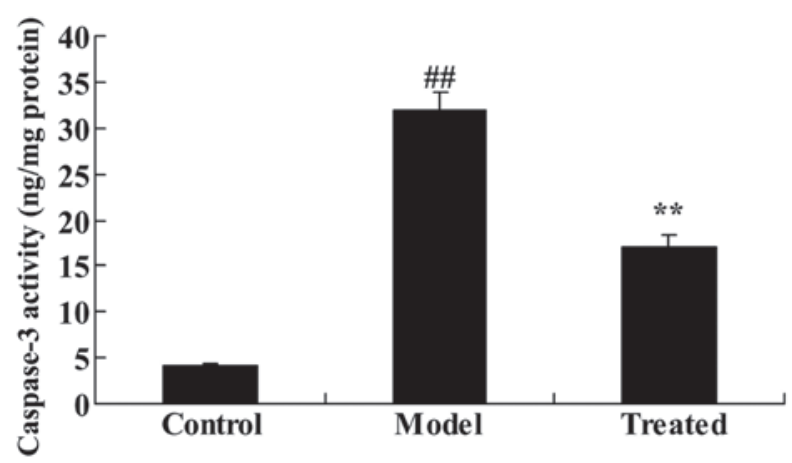

Figure 8. The effect of astaxanthin on the isoflurane-induced caspase- 3 activity in mice organotypic hippocampal cells. ${ }^{\# \#} \mathrm{P}<0.01$ vs the control group and ${ }^{* *} \mathrm{P}<0.01$ vs the model group. Model, isoflurane-treated; treated, astaxanthin + isoflurane-treated.

The effect of astaxanthin reduces the isoflurane-induced caspase-3 activity in mouse organotypic hippocampal cells. Isoflurane treatment significantly induced the caspase- 3 activity compared with the control group ( $\mathrm{P}<0.01 ;$ Fig. 8). Furthermore, supplementary treatment with astaxanthin significantly suppressed the isoflurane-induced caspase- 3 activity compared with the model group $(\mathrm{P}<0.01 ;$ Fig. 8$)$.

Astaxanthin activates the isoflurane-suppressed PI3K/Akt pathway in mouse organotypic hippocampal cells. To explore whether the anti-apoptotic effects of astaxanthin protect against the isoflurane-induced PI3K/Akt pathway activation in organotypic hippocampal cells, p-Akt and Akt protein expression levels were determined with western blotting. The results indicated that isoflurane treatment significantly suppressed the p-Akt/Akt ratio compared with the control group $(\mathrm{P}<0.01$; Fig. 9). In addition, supplementary treatment with astaxanthin significantly activated the isoflurane-suppressed PI3K/Akt pathway, compared with the model group $(\mathrm{P}<0.01$; Fig. 9).

Downregulation of the PI3K/Akt pathway reduces the effect of astaxanthin and protects against isoflurane-induced neuroapoptosis. To further assess the effect of the PI3K/Akt pathway, LY294002 $(20 \mu \mathrm{M})+$ isoflurane were administered to the organotypic hippocampal cells and they were incubated for 24 h. Administration of LY294002 significantly inhibited the 

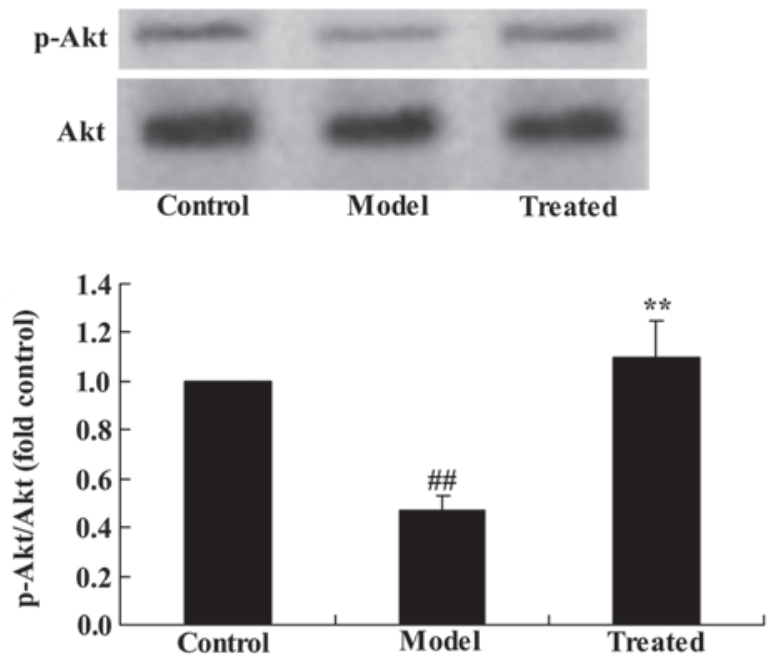

Figure 9. Astaxanthin reduces the isoflurane-induced caspase-3 activity in mice organotypic hippocampal cells. ${ }^{\# \#} \mathrm{P}<0.01$ vs the control group and ${ }^{* *} \mathrm{P}<0.01$ vs the model group. $\mathrm{p}-\mathrm{Akt}$, phosphorylated protein kinase $\mathrm{B}$; model, isoflurane-induced; treated, astaxanthin-treated.

effect of astaxanthin on the p-Akt/Akt ratio (Fig. 10A and B). Furthermore, treatment with LY294002 significantly reduced the effect of isoflurane treatment on caspase-3 activity compared with the model group (Fig. 10C).

\section{Discussion}

Neuronal apoptosis resulting from the use of inhalation anesthetics is detected in the early and late stages of nervous system development (7). Inhalation anesthetics do not exert obvious toxic effects in the late stage (12), due to the difficulty in inducing neuronal damage (13). In addition, in the early stage, particularly the stage of rapid formation of synapses, inhalation anesthetics may activate the cell death pathway (14), and lead to disruption of synaptic remodeling and differentiation and maturation of axons (15). Neurotoxicity due to calcineurin $(\mathrm{CN})$ inhibitors has a significant impact on the healing and quality of life of patients (15). The neurotoxicity of $\mathrm{CN}$ inhibitors has complex mechanisms and clinical manifestations (16). Elucidation of the mechanisms of neurotoxicity, and development in clinical prevention remain emphases $(12,13)$. The present study demonstrated that astaxanthin significantly reduced the isoflurane-induced brain damage. Franceschelli et al (17) demonstrated that astaxanthin protects against stimulation of U937 cells with lipopolysaccharide, reducing $\mathrm{O}_{2}$-production through suppression of oxidative stress. Lu et al (18) demonstrated that astaxanthin protects against neuron loss through suppression of oxidative stress in the adult rat hippocampus. The results of these studies suggest that astaxanthin may reduce isoflurane-induced neuroapoptosis.

During the developmental process of neurons, the formation of new neurons is cross-linked with each other between networks (16). Isoflurane interferes with the formation of neuronal networks during the developmental process to hinder the maturation and differentiation of neurons (19). The effect of inhalation anesthetics on the developing nervous system is not limited to neural degeneration and induced

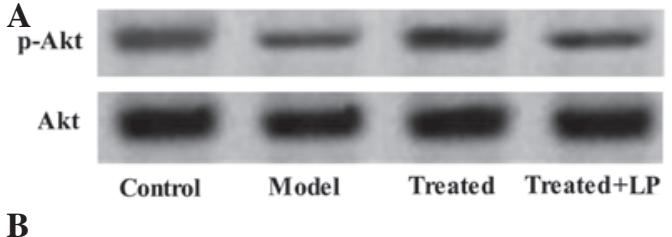

B

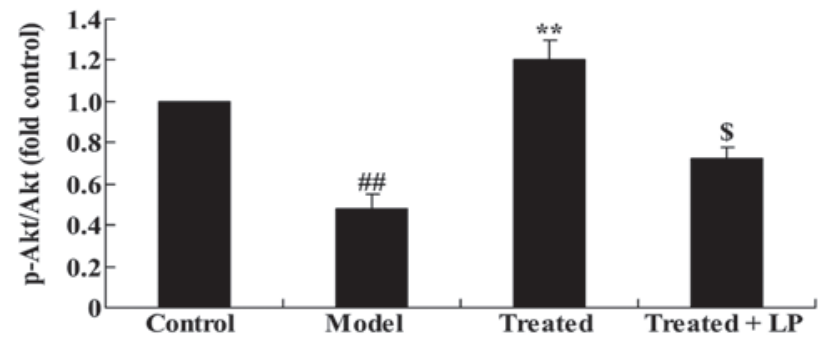

C

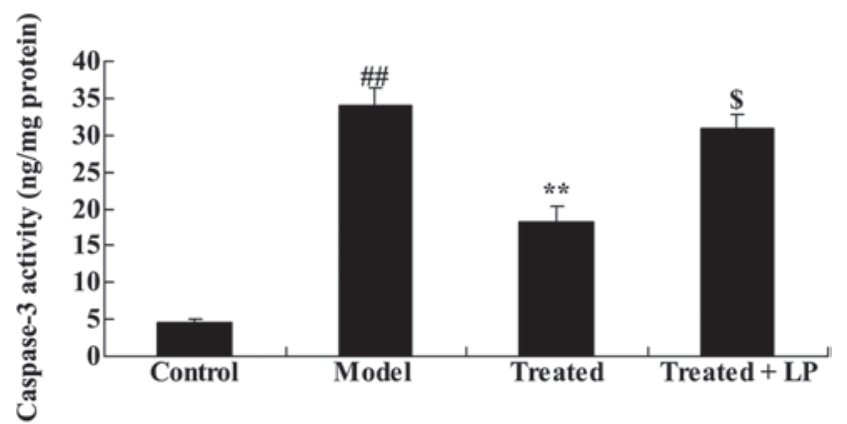

Figure 10. p-Akt/Akt ratio and activity of caspase-3. The protein expression levels of Akt and p-Akt were analyzed by (A) western blotting, followed by (B) densitometry. (C) Caspase-3 activity was significantly increased in the model group and reduced in the treated and treated + LP treated groups. ${ }^{\# \#} \mathrm{P}<0.01$ vs. the control group; ${ }^{* *} \mathrm{P}<0.01$ vs. the model group; ${ }^{\$} \mathrm{P}<0.01$ vs. the treated group. p-Akt, phosphorylated-Akt; model, isoflurane-induced; treated, astaxanthin-treated; LP, LY294002.

neuroapoptosis (20). Other mechanisms are involved in the toxic isoflurane-induced effect. Numerous studies confirm that pretreatment with isoflurane for a short period of time results in a protective effect $(16,21,22)$. If the time of exposure is extended, there can be toxic effects. A previous study suggested that pretreatment with isoflurane is associated with neuroprotection and cardioprotection (23). Therefore, it is suggested that isoflurane has neuroprotective effects and neurotoxic effects simultaneously (24). The effect of isoflurane is dependent on its concentration, exposure time and the patient tolerance (24). The results of the present study demonstrated that astaxanthin significantly inhibited the isoflurane-induced caspase-3 activity in vivo and in vitro. These results are consistent with those of Lee et al (25) who demonstrated that astaxanthin protects against MPP-induced mitochondrial dysfunction through inhibition of the activation of caspase-3 in vivo and in vitro. Chan et al (26) indicated that the neuroprotective effects of astaxanthin alleviated $\mathrm{H}_{2} \mathrm{O}_{2}$ - or $\mathrm{MPP}^{+}$-induced cell death of PC12 cells. Based on these results, the anti-apoptotic effect of astaxanthin is suggested to serve an important role in the treatment of isoflurane-induced neuroapoptosis.

Neuroapoptosis is an important form of programmed cell death subsequent to anesthesia with isoflurane (27). The activation of signal transduction processes during the early stage of apoptosis is required for neuroapoptosis. An 
early intervention to the signaling pathways may result in suppression of neuroapoptosis and protection against brain damage (23). The PI3K/Akt signaling pathway has been shown to promote cell survival (28). Neuroprotective agents targeting Akt have an effect on neuroapoptosis following anesthesia with isoflurane (7). The PI3K/Akt signaling pathway is activated by stimulation of endogenous and exogenous neurotrophic factors (7). The present study demonstrated that astaxanthin significantly upregulated the PI3K/Akt pathway in the isoflurane-treated rats and mouse hippocampal cells. Furthermore, downregulation of the PI3K/Akt pathway reduced the effects of astaxanthin against isoflurane induced neuroapoptosis in vitro. Guo et al (29) suggested that astaxanthin attenuates early acute kidney injury through the Akt/Bad/Caspases signaling cascade. Li et al (30) indicated that astaxanthin protects the ARPE-19 cells through activation of the PI3K/Akt pathway. In conclusion, the results of the present study indicated that astaxanthin reversed isoflurane-induced neuroapoptosis through activation of the PI3K/Akt signaling pathway, in vivo and in vitro.

\section{References}

1. Johnson SA, Young C and Olney JW: Isoflurane-induced neuroapoptosis in the developing brain of nonhypoglycemic mice. J Neurosurg Anesthesiol 20: 21-28, 2008.

2. Deng M, Hofacer RD, Jiang C, Joseph B, Hughes EA, Jia B, Danzer SC and Loepke AW: Brain regional vulnerability to anaesthesia-induced neuroapoptosis shifts with age at exposure and extends into adulthood for some regions. Br J Anaesth 113 443-451, 2014.

3. Wang W, Chen X, Zhang J, Zhao Y, Li S, Tan L, Gao J, Fang X and Luo A: Glycyrrhizin attenuates isoflurane-induced cognitive deficits in neonatal rats via its anti-inflammatory activity. Neuroscience 316: 328-336, 2016.

4. Brambrink AM, Back SA, Riddle A, Gong X, Moravec MD, Dissen GA, Creeley CE, Dikranian KT and Olney JW: Isoflurane-induced apoptosis of oligodendrocytes in the neonatal primate brain. Ann Neurol 72: 525-535, 2012.

5. Cattano D, Williamson P, Fukui K, Avidan M, Evers AS, Olney JW and Young C: Potential of xenon to induce or to protect against neuroapoptosis in the developing mouse brain. Can J Anaesth 55: 429-436, 2008.

6. Ge HW, Hu WW, Ma LL and Kong FJ: Endoplasmic reticulum stress pathway mediates isoflurane-induced neuroapoptosis and cognitive impairments in aged rats. Physiol Behav 151: 16-23, 2015.

7. Li Y, Zeng M, Chen W, Liu C, Wang F, Han X, Zuo Z and Peng S: Dexmedetomidine reduces isoflurane-induced neuroapoptosis partly by preserving PI3K/Akt pathway in the hippocampus of neonatal rats. PLoS One 9: e93639, 2014.

8. Creeley CE, Dikranian KT, Dissen GA, Back SA, Olney JW and Brambrink AM: Isoflurane-induced apoptosis of neurons and oligodendrocytes in the fetal rhesus macaque brain. Anesthesiology 120: 626-638, 2014.

9. Manunta C: Astaxanthin in insects and other terrestrial arthropods. Nature 162: 298, 1948.

10. Ambati RR, Phang SM, Ravi S and Aswathanarayana RG: Astaxanthin: Sources, extraction, stability, biological activities and its commercial applications - a review. Mar Drugs 12 128-152, 2014.

11. Fassett RG and Coombes JS: Astaxanthin in cardiovascular health and disease. Molecules 17: 2030-2048, 2012.

12. Li Y, Yuan Z, Liu B, Sailhamer EA, Shults C, Velmahos GC, Demoya $M$ and Alam HB: Prevention of hypoxia-induced neuronal apoptosis through histone deacetylase inhibition. J Trauma 64: 863-870; discussion 870-871, 2008.
13. Rivera LR, Thacker M, Pontell L, Cho HJ and Furness JB: Deleterious effects of intestinal ischemia/reperfusion injury in the mouse enteric nervous system are associated with protein nitrosylation. Cell Tissue Res 344: 111-123, 2011.

14. Yel L, Brown LE, Su K, Gollapudi S and Gupta S: Thimerosal induces neuronal cell apoptosis by causing cytochrome $\mathrm{c}$ and apoptosis-inducing factor release from mitochondria. Int $\mathrm{J}$ Mol Med 16: 971-977, 2005.

15. Payette DJ, Xie J, Shirwany N and Guo Q: Exacerbation of apoptosis of cortical neurons following traumatic brain injury in par-4 transgenic mice. Int J Clin Exp Pathol 1: 44-56, 2008.

16. Fujimura M and Usuki F: Methylmercury causes neuronal cell death through the suppression of the TrkA pathway: In vitro and in vivo effects of TrkA pathway activators. Toxicol Appl Pharmacol 282: 259-266, 2015 .

17. Franceschelli S, Pesce M, Ferrone A, De Lutiis MA, Patruno A, Grilli A, Felaco M and Speranza L: Astaxanthin treatment confers protection against oxidative stress in U937 cells stimulated with lipopolysaccharide reducing $\mathrm{O}_{2}$-production. PLoS One 9: e88359, 2014.

18. Lu Y, Xie T, He XX, Mao ZF, Jia LJ, Wang WP, Zhen JL and Liu LM: Astaxanthin rescues neuron loss and attenuates oxidative stress induced by amygdala kindling in adult rat hippocampus. Neurosci Lett 597: 49-53, 2015.

19. Agarwal B, Camara AK, Stowe DF, Bosnjak ZJ and Dash RK: Enhanced charge-independent mitochondrial free $\mathrm{Ca}(2+)$ and attenuated ADP-induced NADH oxidation by isoflurane: Implications for cardioprotection. Biochim Biophys Acta 1817: 453-465, 2012.

20. Zhong Y, Liang Y, Chen J, Li L, Qin Y, Guan E, He D, Wei Y, Xie Y and Xiao Q: Propofol inhibits proliferation and induces neuroapoptosis of hippocampal neurons in vitro via downregulation of $\mathrm{NF}-\kappa \mathrm{B}$ p65 and $\mathrm{Bcl}-2$ and upregulation of caspase-3. Cell Biochem Funct 32: 720-729, 2014.

21. Cheng Y and Levy RJ: Subclinical carbon monoxide limits apoptosis in the developing brain after isoflurane exposure. Anesth Analg 118: 1284-1292, 2014.

22. Makaryus R, Lee H, Yu M, Zhang S, Smith SD, Rebecchi M, Glass PS and Benveniste H: The metabolomic profile during isoflurane anesthesia differs from propofol anesthesia in the live rodent brain. J Cereb Blood Flow Metab 31: 1432-1442, 2011

23. Lang XE, Wang X and Jin JH: Mechanisms of cardioprotection by isoflurane against I/R injury. Front Biosci (Landmark Ed) 18: 387-393, 2013.

24. BaiT,Dong DS and Pei L: Resveratrol mitigates isoflurane-induced neuroapoptosis by inhibiting the activation of the Akt-regulated mitochondrial apoptotic signaling pathway. Int J Mol Med 32: 819-826, 2013.

25. Lee DH, Kim CS and Lee YJ: Astaxanthin protects against MPTP/MPP+-induced mitochondrial dysfunction and ROS production in vivo and in vitro. Food Chem Toxicol 49: 271-280, 2011.

26. Chan KC, Mong MC and Yin MC: Antioxidative and anti-inflammatory neuroprotective effects of astaxanthin and canthaxanthin in nerve growth factor differentiated PC12 cells. J Food Sci 74: H225-H231, 2009.

27. Kajimoto M, Atkinson DB, Ledee DR, Kayser EB, Morgan PG, Sedensky MM, Isern NG, Des Rosiers C and Portman MA: Propofol compared with isoflurane inhibits mitochondrial metabolism in immature swine cerebral cortex. J Cereb Blood Flow Metab 34: 514-521, 2014.

28. Guo LY, Li YM, Qiao L, Liu T, Du YY, Zhang JQ, He WT, Zhao YX and He DQ: Notch2 regulates matrix metallopeptidase 9 via PI3K/AKT signaling in human gastric carcinoma cell MKN-45. World J Gastroenterol 18: 7262-7270, 2012.

29. Guo SX, Zhou HL, Huang CL, You CG, Fang Q, Wu P, Wang XG and Han CM: Astaxanthin attenuates early acute kidney injury following severe burns in rats by ameliorating oxidative stress and mitochondrial-related apoptosis. Mar Drugs 13: 2105-2123, 2015.

30. Li Z, Dong X, Liu H, Chen X, Shi H, Fan Y, Hou D and Zhang X: Astaxanthin protects ARPE-19 cells from oxidative stress via upregulation of Nrf2-regulated phase II enzymes through activation of PI3K/Akt. Mol Vis 19: 1656-1666, 2013. 